\title{
An Efficient Method Based on the Electromagnetic Time Reversal to Locate Faults in Power Networks
}

\author{
Reza Razzaghi, Student Member, IEEE, Gaspard Lugrin, Hossein Manesh, Carlos Romero, \\ Mario Paolone, Senior Member, IEEE, and Farhad Rachidi, Fellow, IEEE
}

\begin{abstract}
This paper presents a new method based on the electromagnetic time-reversal (EMTR) theory for locating faults in power networks. The applicability of the EMTR technique to locate faults is first discussed. Using the classical transmission-line equations in the frequency domain, analytical expressions are derived to infer the location of the fault. The accuracy of the proposed method is then discussed in relation to the number of observation points adopted to record the fault-originated electromagnetic transients. Then, this paper illustrates the extension of the proposed method to the time domain. The experimental validation of the proposed method is presented by making reference to a reduced-scale coaxial cable system where real faults are hardware-emulated. Finally, the application of the proposed EMTRbased fault-location method to Electromagnetic Transients Program-simulated cases is presented. The simulated test cases are: a mixed overhead/coaxial cable transmission system and the IEEE 34-bus distribution test feeder. Compared to other transient-based fault-location techniques, the proposed method presents a number of advantages, namely, its straightforward applicability to inhomogeneous media (mixed overhead and coaxial power cable lines), the use of a single observation (measurement) point, and robustness against fault type and fault impedance.
\end{abstract}

Index Terms-Electromagnetic time reversal, fault location, fault transients, power systems protection.

\section{INTRODUCTION}

$\mathbf{T}$ HE fault-location functionality is an important online process required by power systems operation. It has a large influence on the security and quality of supply.

In transmission networks, this functionality is needed for the identification of the faulted line and the adequate reconfiguration of the network to anticipate severe cascading consequences. In distribution networks, fault location is more associated with the quality of service in terms of duration of interruptions when permanent faults occur. Still, with reference to distribution networks, the increasing use of distributed generation calls for accurate and fast fault-location procedures aimed at minimizing the network service restoration time and, consequently, minimizing the unsupplied power.

As summarized in [1] and [2], various procedures for fault-location assessment have been proposed for transmission and distribution power networks and, in this respect, two main cate-

Manuscript received September 06, 2012; revised January 24, 2013; accepted February 26, 2013. Date of publication April 16, 2013; date of current version June 20, 2013. Paper no. TPWRD-00932-2012.

The authors are with the Distributed Electrical Systems Laboratory (DESL) and Electromagnetic Compatibility Laboratory (EMC), École Polytechnique Fédérale de Lausanne (EPFL), Lausanne 1015, Switzerland (e-mail: reza.razzaghi@epfl.ch; gaspard.lugrin@epfl.ch; hossein.manesh@epfl.ch; carlos.romero@epfl.ch; mario.paolone@epfl.ch; farhad.rachidi@epfl.ch).

Digital Object Identifier 10.1109/TPWRD.2013.2251911 gories can be identified: 1) methods that analyze prefault and postfault voltage/current phasors (e.g., [3]-[5]) and 2) methods that analyze fault-originated electromagnetic transients of currents and/or voltages, (i.e., travelling waves generated by the fault itself, for example, [6]-[14]).

As discussed in [2], with reference to the case of distribution networks, typical methods used these days are based on the estimation of the postfault impedance observed in measurement points usually located in primary substations. With the hypothesis of having a fully passive power system, such an estimation could provide useful information to locate the fault when compared with the line impedance. However, the presence of other sources (e.g., associated with the increasing penetration of dispersed generation) can largely affect the accuracy of these procedures. For these reasons, procedures that belong to the second of the aforementioned categories may be less influenced by the presence of dispersed generation. This is because postfault high-frequency electromagnetic transients taking place within the first few milliseconds after the fault are associated with the travelling waves originated by the fault itself. Therefore, these transients are not influenced by the industrial frequency power injections of distributed sources. The major drawbacks of this second category of methods are: 1) they require an assessment between the number of observation points versus the number of possible multiple fault-location solutions and 2) they require large bandwidth measuring systems.

Within this context and by referring to the second of the aforementioned categories, this paper aims at investigating the possibility of applying the theory of time reversal to the problem of fault location. This method was developed first in the field of acoustics [15]-[18] and was more recently applied to electromagnetics (e.g., [19]-[22]). In what follows, we will make reference to the time-reversal process applied to electromagnetic transients using the acronym electromagnetic time reversal (EMTR).

The basic idea of the EMTR is to take advantage of the reversibility in time of the wave equation. The transients observed in specific observation points of the system are time-reversed and transmitted back into the system. The time-reversed signals are shown to converge to the source (fault) location. The EMTR presents several advantages, namely: 1) applicability to inhomogeneous media [15] and 2) efficiency for systems bounded in space [18] and characterized by complex topology (in our case, networks with multiple termination).

In the literature related to fault location in wired networks, a method based on the use of the so-called "matched-pulse reflectometry," essentially based on the reflectometry approach and embedding some of the properties of the EMTR, has recently been presented in [23]. Another method based on the 
time reversal, called decomposition of the time-reversal operator (DORT) [24], was also proposed to locate soft faults in wired networks [25].

In a previous study [2], the authors of this paper have presented a preliminary discussion on the applicability of the EMTR technique to locate faults in a single-conductor transmission line using multiple observation points. This paper aims at extending the applicability of such a fault-location technique to the case of a single observation point and multiconductor, inhomogeneous transmission lines (for example, composed of a number of overhead lines and coaxial cables). In addition, this paper presents: 1) an experimental validation of the proposed fault-location method by making use of a reduced-scale coaxial cable setup with hardware-emulated faults; 2) an illustration of the proposed method's applicability to complex distribution networks (i.e., the one represented by the IEEE test distribution feeders); and 3) an analysis of the robustness of the method against the fault impedance and type.

The structure of this paper is as follows. Section II describes the basic aspects of EMTR theory.Section III illustrates the main characteristics of electromagnetic transients originated by faults in power systems. In Section IV, the applicability of the EMTR technique to locate faults is first presented and then, using transmission-line equations in the frequency domain, analytical expressions are derived, permitting inference of the location of the fault. In the same section, the time-domain implementation of the EMTR-based fault-location technique is also illustrated. Section V reports the experimental validation of the proposed methodology using a reduced-scale model. Finally, Section VI illustrates the application of the proposed method to two cases: 1) an inhomogeneous network composed of mixed overhead coaxial cable lines and 2) a distribution network composed of the IEEE 34-bus test feeder. In both cases, different fault types and fault impedances have been considered. Section VII concludes this paper by addressing the final remarks on the performance of the proposed method.

\section{BASIC CONCEPT OF THE EMTR TECHNIQUE}

In this section, we examine the properties of the transmissionline wave equations under time reversal[2]. The time-reversal operator corresponds to the change of the sign of the time,that is, to the following transformation:

$$
t \mapsto-t
$$

An equation is defined as "time-reversal invariant" "if it is invariant under the application of the time-reversal operator. The voltage wave equation for a multiconductor, lossless transmission line reads

$$
\frac{\partial^{2}}{\partial x^{2}} \mathbf{U}(x, t)-\mathbf{L}^{\prime} \mathbf{C}^{\prime} \frac{\partial^{2}}{\partial t^{2}} \mathbf{U}(x, t)=0
$$

where $\mathbf{U}(x, t)$ is a vector containing the phase voltages at position $x$ and time $t$, and $\mathbf{L}^{\prime}$ and $\mathbf{C}^{\prime}$ are the per-unit-length matrices of the inductance and capacitance of the line, respectively. Time reversing (2) yields

$$
\frac{\partial^{2}}{\partial x^{2}} \mathbf{U}(x,-t)-\mathbf{L}^{\prime} \mathbf{C}^{\prime} \frac{\partial^{2}}{\partial t^{2}} \mathbf{U}(x,-t)=0 .
$$

Therefore, if $\mathbf{U}(x, t)$ is a solution of the wave equation, then $\mathbf{U}(x,-t)$ is a solution too. In other words, as described in [16]-[18] for ultrasonic waves, the wave equation is invariant under a time-reversal transformation if there is no absorption during propagation in the medium. In our specific application, this hypothesis is satisfied if the transmission line is lossless. However, since power network transmission lines are generally characterized by small values for longitudinal resistance, the applicability of EMTR to this case could also be considered. This point will be further discussed in Sections V and VI

In practical implementations, a signal $s(x, t)$ is necessarily measured only during a finite period of time from an initial time selected here as the origin $t=0$ to a final time $t=T$, where $T$ is the duration of the signal. To make the argument of the time-reversed variables be positive for the duration of the signal, we will add, in addition to time reversal, a time delay $T$ [26]

$$
s(x, t) \mapsto s(x, T-t) .
$$

It is worth noting that although the EMTR is defined in the time domain, it can also be applied in the frequency domain using the following equivalence:

$$
f(\vec{r},-t) \leftrightarrow \mathrm{F}^{*}(\vec{r}, \omega)
$$

where $\mathrm{F}(\vec{r}, \omega)$ is the Fourier transform of $f(\vec{r}, t)$ and $*$ denotes the complex conjugate.

\section{Electromagnetic Transients Associated With FAULTS IN POWER SYSTEMS}

A fault event in a power system can be associated with an injection into the power system itself of a step-like wave triggered by the fault occurrence. The fault-generated wave travels along the lines of the network and gets reflected at the line extremities which are characterized by reflection coefficients whose values depend on the line surge impedance (characteristic impedance) and the input impedances of the connected power components. In particular, the line extremities can be grouped into three categories, namely: line terminals with power transformers, junctions to other lines, and the fault location. As discussed in [14] and [27], for each of these boundary conditions, the following assumptions can be reasonably made:

- extremities where a power transformer is connected can be assumed, for the traveling waves, as open circuits, and therefore the relevant voltage reflection coefficient is close to +1 ; indeed, fault-originated travelling waves are characterized by a spectrum with high-frequency components for which the input impedance of power transformers is generally dominated by a capacitive behavior with capacitances values in the order of few hundreds of pF (e.g., [27]);

- extremities that correspond to a junction between more than two lines are characterized by a negative reflection coefficient;

- fault location: the reflection coefficient of the extremity where the fault is occurring is close to -1 , as the fault impedance can be assumed to be significantly lower than the line surge impedance.

With the aforementioned assumptions and for a given network topology, it is possible to determine a certain number of 


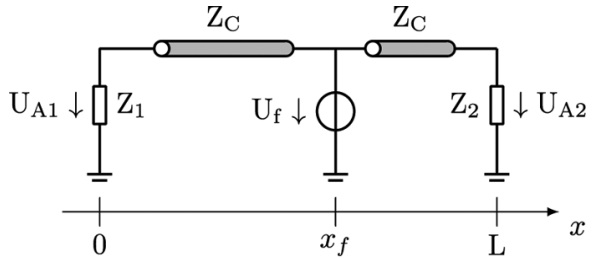

Fig. 1. Simplified representation of the postfault line configuration.

paths, each one delimited between two extremities. An observation point at which voltage or current waveforms are measured will observe a superposition of waves that are traveling along the various paths [28].

Therefore, we can conclude that the domain of application of any fault-location method belonging to the second category described in Section I is formed by a mono-dimensional space (associated to the line longitudinal coordinate $x$ ) with given boundary conditions.

\section{Application of the EMTR Method to Fault Location}

The application of the EMTR to locate faults in a power network will be based on the following steps: 1) measurement of the fault-originated electromagnetic transient in a single observation point; 2) simulation of the back-injection of the time-reversed measured fault signal for different guessed fault locations and using the network model; and (iii) assessment of the fault location by determining, in the network model, the point characterized by the largest energy concentration associated with the back-injected time-reversed fault transients. These aspects will be better clarified in the following sections.

In what follows, we illustrate the analytical aspects related to the proposed EMTR-based fault-location method. As described in [15]-[21], one of the main hypotheses of the EMTR method is that the topology of the system needs to remain unchanged during the transient phenomenon of interest. Fault transients in power networks do not satisfy such a condition as the presence of the fault itself involves a change in the network topology when the fault occurs (i.e., at $t=t_{f}$ ). However, for reversed times $t$ such that $t<T-t_{f}$, EMTR is still applicable if the guessed fault is considered at the correct location. On the other hand, for a guessed location that does not coincide with the real one, time-reversal invariance does not hold. As a result of this property, time-reversed back-propagated signals will combine constructively to reach a maximum at the correct fault location. This property will be validated in the next sections using both experimental measurements and simulation test cases.

In order to provide a more straightforward use of the EMTR technique, we will make reference to a single-conductor overhead lossless transmission line (see Fig. 1) of length $L$. The line parameters may refer to a typical overhead transmission line. In particular, the surge impedance (characteristic impedance) is in the order of a few hundred ohms.

We assume that at both line extremities, power transformers are connected. Therefore, as discussed in Section III, they are represented by means of high input impedances $\left(Z_{1}\right.$ and $Z_{2}$ in Fig. 1). The fault coordinate is $x_{f}$ and fault transient waveforms are assumed to be recorded either at one end or at the two ends of the line. Since the line model is lossless, the damping of the transients is provided only by the fault impedance, if any, and the high terminal impedances $Z_{1}$ and $Z_{2}$.

Finally, as the analyzed fault transients last for only a few milliseconds, we assume that the prefault condition of the line is characterized by a constant value of voltage all along the line length $(0 \leq x \leq L)$.

\section{A. Frequency-Domain Expressions of Electromagnetic Transients Generated By the Fault}

The aim of this subsection is to analytically describe the behavior of the line response after a fault. In order to express analytically the line response, the problem is formulated in the frequency domain. To specify the boundary conditions of the two line sections of Fig. 1, namely, for $0 \leq x \leq x_{f}$ and $x_{f} \leq x \leq L$. we can define reflection coefficients at $x=0$ $(i=1$ of Fig. 1$)$ and $x=L(i=2$ of Fig. 1$)$ as

$$
\rho_{i}=\frac{Z_{i}-Z_{C}}{Z_{i}+Z_{C}} ; \quad i=1,2 .
$$

Without losing generality, coefficients $\rho_{i}$ in (6) could be assumed as frequency independent within the considered short observation time.

Concerning the boundary condition at the fault location, we assume to represent it by means of a voltage source $\mathrm{U}_{\mathrm{f}}(\omega)$ located at $x=x_{f}$.

For the sake of abstraction, we represent the fault by means of an ideal voltage source with zero internal impedance that, as a consequence, represents a solid fault. Therefore, the voltage reflection coefficient in this point of the line is $\rho_{f}=-1$. In addition, in view of the lossless line assumption, the line propagation constant, $\gamma$, is purely imaginary, namely: $\gamma=\mathrm{j} \beta$, with $\beta=\omega / c$. The analytical expressions of voltages observed at the line terminals $x=0$ and $x=L$ in the frequency domain read

$$
\begin{aligned}
& \mathrm{U}_{\mathrm{A} 1}(\omega)=\mathrm{U}(0, \omega)=\frac{\left(1+\rho_{1}\right) \mathrm{e}^{-\gamma x_{f}}}{1+\rho_{1} \mathrm{e}^{-2 \gamma x_{f}}} \mathrm{U}_{\mathrm{f}}(\omega) \\
& \mathrm{U}_{\mathrm{A} 2}(\omega)=\mathrm{U}(L, \omega)=\frac{\left(1+\rho_{2}\right) \mathrm{e}^{-\gamma\left(L-x_{f}\right)}}{1+\rho_{2} \mathrm{e}^{-2 \gamma\left(L-x_{f}\right)}} \mathrm{U}_{\mathrm{f}}(\omega) .
\end{aligned}
$$

Note that the effect of the ground losses can be represented as an additional frequency-dependent longitudinal impedance [29]. However, except for the case of distributed exciting sources (such as those produced by a nearby lightning discharge), ground losses can be disregarded for typical overhead power lines [30].

In the two following subsections, we will derive EMTRbased analytical expressions that allow us to infer the location of the fault, for both cases of multiple and single observation points.

\section{B. Frequency-Domain Application of EMTR by Using Two Observation Points at Each Line Terminal}

In principle, a number of observation points at which transient signals initiated by the fault are measured could be used to apply the EMTR technique. In the first step,it is assumed that two observation points at both ends of the line are used.

Equation (7) and (8) provide the frequency-domain expressions of fault-originated voltages at two observation points located at the line terminals. In agreement with the EMTR, 


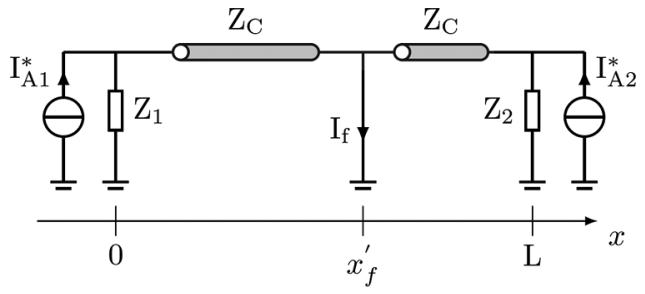

Fig. 2. Representation of the EMTR applied to the single-line model of Fig. 1.

we can replace these observation points with two sources each one imposing the time-reversed voltage fault transient, namely, $\mathrm{U}_{\mathrm{A} 1}^{*}(\omega)$ and $\mathrm{U}_{\mathrm{A} 2}^{*}(\omega)$, where * denotes the complex conjugate. Since the reflection coefficients $\rho_{1}$ and $\rho_{2}$ are almost equal to 1 , it is preferable to use the Norton equivalents as

$$
\begin{aligned}
& \mathrm{I}_{\mathrm{A} 1}^{*}=\frac{\mathrm{U}_{\mathrm{A} 1}^{*}(\omega)}{Z_{1}} \\
& \mathrm{I}_{\mathrm{A} 2}^{*}=\frac{\mathrm{U}_{\mathrm{A} 1}^{*}(\omega)}{Z_{2}}
\end{aligned}
$$

where $\mathrm{I}_{\mathrm{A} 1}^{*}(\omega)$ and $\mathrm{I}_{\mathrm{A} 2}^{*}(\omega)$ are the injected currents as shown in Fig. 2.

Since the location of the fault is the unknown of the problem, we will place it at a generic location $x_{\mathrm{f}}^{\prime}$. The contributions in terms of currents at the unknown fault location $x_{\mathrm{f}}^{\prime}$ coming from the first and the second time-reversed sources $\mathrm{I}_{\mathrm{A} 1}^{*}(\omega)$ and $\mathrm{I}_{\mathrm{A} 2}^{*}(\omega)$ are given, respectively, by

$$
\begin{aligned}
& \mathrm{I}_{\mathrm{f} 1}\left(x_{f}^{\prime}, \omega\right)=\frac{\left(1+\rho_{1}\right) \mathrm{e}^{-\gamma x_{f}^{\prime}}}{1+\rho_{1} \mathrm{e}^{-2 \gamma x_{f}^{\prime}}} \mathrm{I}_{\mathrm{A} 1}^{*}(\omega) \\
& \mathrm{I}_{\mathrm{f} 2}\left(x_{f}^{\prime}, \omega\right)=\frac{\left(1+\rho_{2}\right) \mathrm{e}^{-\gamma\left(L-x_{f}^{\prime}\right)}}{1+\rho_{2} \mathrm{e}^{-2 \gamma\left(L-x_{f}^{\prime}\right)}} \mathrm{I}_{\mathrm{A} 2}^{*}(\omega) .
\end{aligned}
$$

Introducing (7)-(10) into (11) and (12), we obtain

$$
\begin{aligned}
\mathrm{I}_{\mathrm{f} 1}\left(x_{f}^{\prime}, \omega\right)= & \frac{\left(1+\rho_{1}\right)^{2} \mathrm{e}^{-\gamma\left(x_{f}^{\prime}-x_{f}\right)}}{Z_{1}\left(1+\rho_{1} \mathrm{e}^{-2 \gamma x_{f}^{\prime}}\right)\left(1+\rho_{1} \mathrm{e}^{+2 \gamma x_{f}}\right)} \mathrm{U}_{\mathrm{f}}^{*}(\omega)(13) \\
\mathrm{I}_{\mathrm{f} 2}\left(x_{f}^{\prime}, \omega\right)= & \frac{\left(1+\rho_{2}\right)^{2} \mathrm{e}^{-\gamma\left(x_{f}^{\prime}-x_{f}\right)}}{Z_{2}\left(1+\rho_{2} \mathrm{e}^{-2 \gamma\left(L-x_{f}^{\prime}\right)}\right)\left(1+\rho_{2} \mathrm{e}^{+2 \gamma\left(L-x_{f}\right)}\right)} \\
& \times \mathrm{U}_{\mathrm{f}}^{*}(\omega) .
\end{aligned}
$$

Therefore, we can derive a closed-form expression for the total current flowing through the guessed fault location $x_{\mathrm{f}}^{\prime}$

$$
\mathrm{I}_{\mathrm{f}}\left(x_{f}^{\prime}, \omega\right)=\mathrm{I}_{\mathrm{f} 1}\left(x_{f}^{\prime}, \omega\right)+\mathrm{I}_{\mathrm{f} 2}\left(x_{f}^{\prime}, \omega\right) .
$$

In what follows, we will make use of (15) to show the capability of the EMTR to converge the time-reversed injected energy to the fault location.

Let us make reference to a line characterized by a total length $L=10 \mathrm{~km}$ and let us assume a fault occurring at $x_{\mathrm{f}}=8 \mathrm{~km}$. The line is characterized by terminal impedances $Z_{1}=Z_{2}=$ $100 \mathrm{k} \Omega$ and, for the fault, we assume $U_{f}=1 / j \omega \mathrm{V} /(\mathrm{rad} / \mathrm{s})$. By varying $x_{\mathrm{f}}^{\prime}$ from 0 to $L$, it is possible to compute the current at the guessed fault locations using (15). The dotted line in Fig. 3 shows the normalized energy of $I_{\mathrm{f}}$ (where the normalization has been implemented with respect to the maximum signal energy value of $I_{\mathrm{f}}$ for all the guessed fault locations) within a frequency spectrum ranging from dc to $1 \mathrm{MHz}$. From Fig. 3, it is clear that

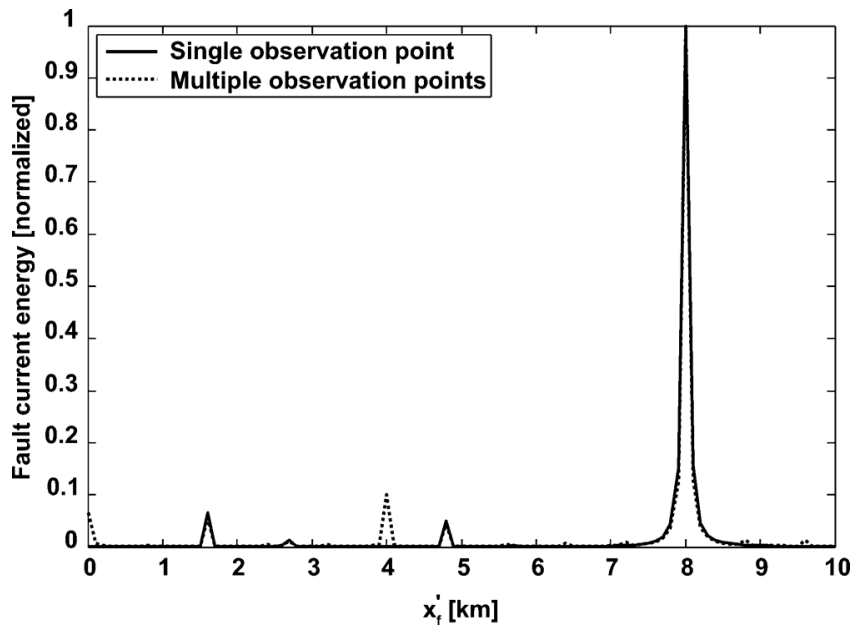

Fig. 3. Normalized energy of the fault current signal as a function of the guessed fault location $x_{\mathrm{f}}^{\prime}$ with single (solid line) and multiple (dashed line) observation points. The real fault location is $x_{\mathrm{f}}=8 \mathrm{~km}$.

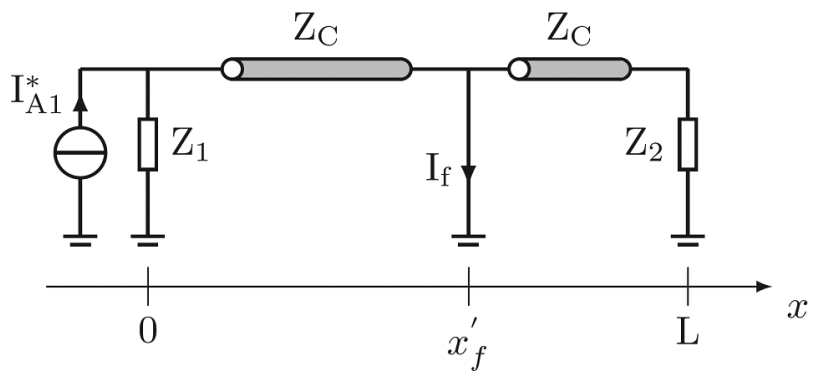

Fig. 4. Representation of the EMTR applied to the single-line model of Fig. 1 where a single observation point is placed at the beginning of the line $(x=0)$.

the energy of $\mathrm{I}_{\mathrm{f}}\left(x_{f}^{\prime}, \Omega\right)$ reaches its maximum when the guessed fault location coincides with the real one.

\section{Frequency-Domain Application of the EMTR by Using One Observation Point at One of the Line Terminals}

One of the main problems in power systems protection, in general, is the limited number of observation points where measurement equipment can be placed. In addition, fault-location methods require, in principle, time synchronization between the measurements. (In other words, the measurement systems located at both ends of the lines should be capable of time-stamping the transients by using the universal time coordinate (UTC).)

Therefore, the demonstration that the EMTR-based fault-location method could be applied also for the case of a single observation point, is of importance.

To this end, let us assume to observe the fault-originated electromagnetic transients only at one location, namely, at the line left terminal. The network schematic in the time-reversal state will be the one in Fig. 4.

By making reference to the configuration of the previous case, we can extend the procedure to the case where only one injecting current source $\left(\mathrm{I}_{\mathrm{A} 1}\right)$ is considered. In particular, we can derive from (9) the fault current at the guessed fault location $x_{\mathrm{f}}^{\prime}$ as follows:

$$
I_{f}\left(x_{f}^{\prime}, \omega\right)=I_{f 1}\left(x_{f}^{\prime}, \omega\right)
$$




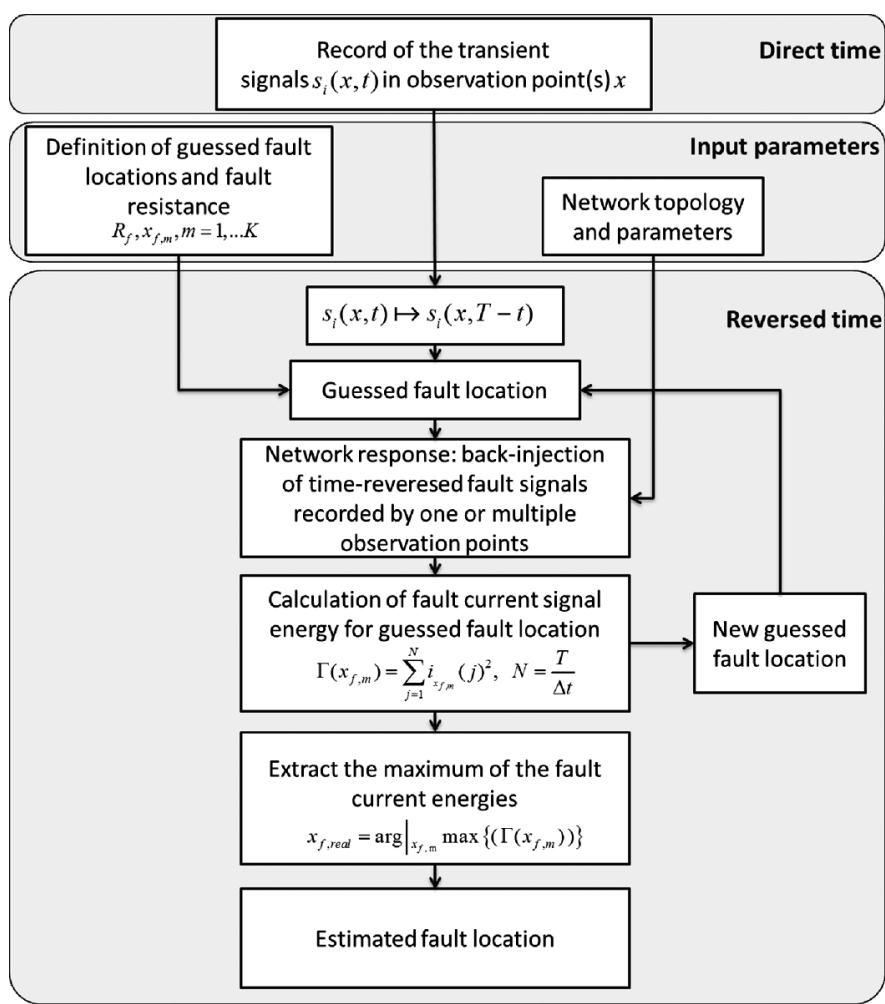

Fig. 5. Flow-chart of the proposed fault-location method.

Fig. 3 shows the normalized energy of $\mathrm{I}_{\mathrm{f}}$ concerning both cases of one and two observation points within a frequency spectrum ranging from dc to $1 \mathrm{MHz}$.

It can be noted that the energy of $\mathrm{I}_{f}\left(x_{f}^{\prime}, \omega\right)$ is maximum when the guessed fault location is equal to the real one even for the case of a single observation point. From Fig. 3, it can be further observed that fault current energy curves feature additional small peaks that correspond to incorrect fault location, although the correct one can still be properly identified. In addition, the two curves (corresponding, respectively, to one and to two observation points) provide the correct fault location with negligible location differences.

\section{Time-Domain EMTR-Based Fault-Location Algorithm}

In the previous subsection, we have inferred closed-form expressions of the fault current as a function of the guessed fault location. The purpose of this subsection is to extend the proposed method to realistic time-domain cases.

The flowchart shown in Fig. 5, illustrates the step-by-step fault-location procedure proposed in this study. As can be seen, the proposed procedure, similarly to other methods proposed in the literature (e.g., [8], [9]), requires the knowledge of the network topology as well as its parameters. Such knowledge is used to build a corresponding network model where the lines are represented, for instance, by using constant-parameters line models [31]. Then, we assume to record fault transients $s_{\mathrm{i}}(t)$ (with $i=1,2,3$ for a three-phase system) at a generic observation point located inside the part of the network with the same voltage level comprised between transformers.
The transient signal initiated by the fault is assumed to be recorded within a specific time window, namely

$$
s_{i}(t), t \in\left[t_{f}, t_{f}+T\right]
$$

where $t_{f}$ is the fault triggering time, and $T$ is the recording time window large enough to damp out $s_{\mathrm{i}}(t)$.

The unknowns of the problem are the fault type, location, and impedance. Concerning the fault type, we assume that the fault-location procedure will operate after the relay maneuver. Therefore, the single or multiphase nature of the fault is assumed to be known. Concerning the fault location, we assume a set of apriori locations $x_{f, m}, m=1, \ldots K$ for which the EMTR procedure is applied.

Concerning the fault impedance, for all the guessed fault locations, an apriori value of the fault resistance, $R_{x f}$, is assumed. As it will be shown in the section containing the application examples (Section VI), different guessed values of $R_{x f}$ do not affect the fault-location accuracy.

Therefore, the recorded signal is reversed in time (1) and back-injected from one or multiple observation points into the system for each $x_{f, m}$. As discussed in Section II, in order to make the argument of the time-reversed variables be positive for the duration of the signal, we add, in addition to time reversal, a time delay equal to the duration of the recording time $T$

$$
\begin{aligned}
& \hat{t}=\left(T+t_{f}\right)-t \\
& \bar{s}(\hat{t}) \hat{t} \in[0, T] .
\end{aligned}
$$

As shown in Fig. 5, for each of the guessed fault locations, we can compute the energy of the signal that corresponds to the currents flowing through the guessed fault location as

$$
\Gamma\left(x_{f, m}\right)=\sum_{j=1}^{N} i_{x_{f, m}}^{2}(j)^{2}, T=N \Delta t
$$

where $N$ is the number of samples and $\Delta t$ is the sampling time. According to the EMTR method presented in the previous subsection, the energy given by (20) is maximized at the real fault location. Thus, the maximum calculated signal energies will indicate the real fault point

$$
x_{\mathrm{f}, \text { real }}=\left.\arg \right|_{x_{f, \mathrm{~m}}} \max \left\{\left(\Gamma\left(x_{f, m}\right)\right)\right\} .
$$

\section{EXPERIMENTAL VALIDATION}

In this section, the experimental validation of the proposed method is presented by making reference to a reduced-scale coaxial cable system. Such a system has been realized by using standard RG-58 and RG-59 coaxial cables where real faults were hardware-emulated.

The topologies adopted to carry out the experimental validation are shown in Fig. 6. As seen on the figure, the first topology corresponds to a single transmission line while the second one corresponds to a T-shape network where the various branches are composed of both RG-58 and RG-59 cables (i.e., each branch has a different surge impedance but the same propagation speed). Fig. 6 shows also the guessed fault locations 


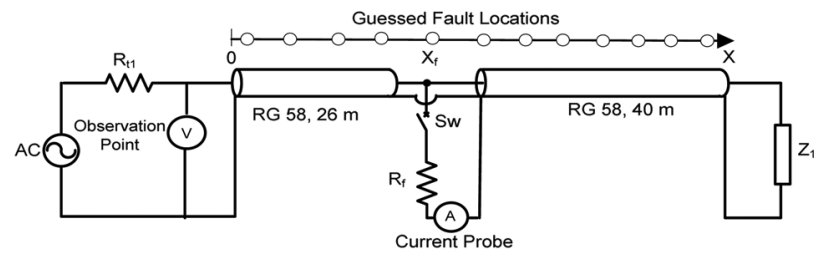

(a)

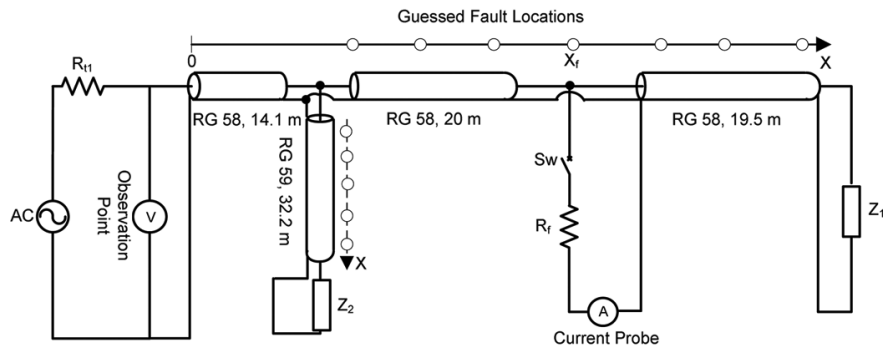

(b)

Fig. 6. Topologies adopted for the reduced-scale experimental setup. (a) Single transmission-line configuration (RG-58 coaxial cable). (b) T-shape network made of RG-58 and RG-59 coaxial cables.

at which the current flowing through the fault was measured. For each considered topology, transients generated by the fault are recorded at one observation point, shown also on Fig. 6. The fault-originated transients were measured by means of a 12-b oscilloscope (LeCroy Waverunner HRO 64Z) operating at a sampling frequency of $1 \mathrm{GSamples/s.} \mathrm{For} \mathrm{the} \mathrm{direct} \mathrm{time,} \mathrm{the}$ oscilloscope directly records voltages at the shown observation points marked in Fig. 6(a) and (b). For the reversed time, the current at each guessed fault location was measured by using a 2877 Pearson current probe characterized by a transfer impedance of $1 \Omega$ and an overall bandwidth of $300 \mathrm{~Hz}-200 \mathrm{MHz} .{ }^{1}$ It is worth observing that the switching frequencies for the adopted reduced-scale systems are in the order of few $\mathrm{MHz}$. The time-reversed transient waveforms were generated by using a 16-b arbitrary waveform generator (LeCroy ArbStudio 1104) operating at a sampling frequency of $1 \mathrm{GSamples/s}$ (the same adopted to record the fault-originated waveforms). The lines were terminated by high impedances $\left(Z_{1}\right.$ and $Z_{2}$ equal to $\left.1 M \Omega\right)$ and the voltage source injecting the time-reversed signal was connected to the line through a lumped resistance of $R=4.7 \mathrm{k} \Omega$ in order to emulate, in a first approximation, the high-input impedance of power transformers with respect to fault transients.

The faults were generated at an arbitrary point of the cable network. They were realized by a short circuit between the coaxial cable shield and the inner conductors. It is important to underlie that these types of faults excite the shield-to-inner conductor propagation mode that is characterized, for the adopted coaxial cables, by a propagation speed of $65.9 \%$ of the speed of light $c$. It is worth noting that the limited lengths of the reduced-scale cables (i.e., tens of meters) involve propagation times on the order of tens to hundreds of nanoseconds. Such a peculiarity requires that the fault emulator needs to be able to change its status in a few nanoseconds in order to correctly emulate the fault. The chosen switch was a high-speed

\footnotetext{
${ }^{1}$ It is worth observing that the switching frequencies for the adopted reduced scale systems are in the order of few $\mathrm{MHz}$.
}

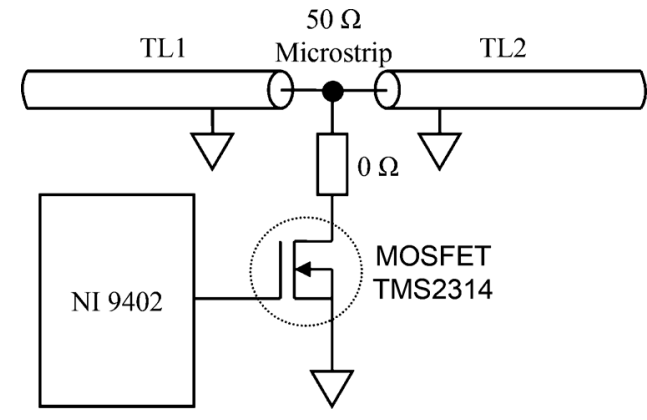

Fig. 7. Schematic representation of the MOSFET-emulated fault adopted in the reduced-scale experimental setup.

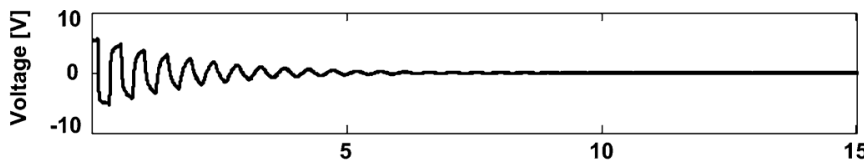

(a)

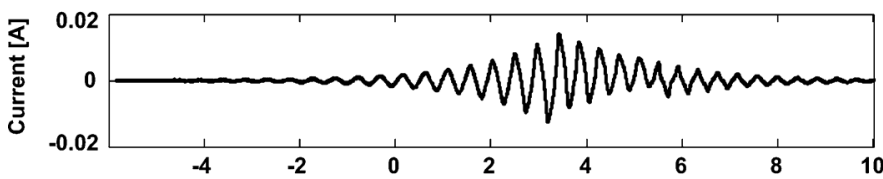

(b)

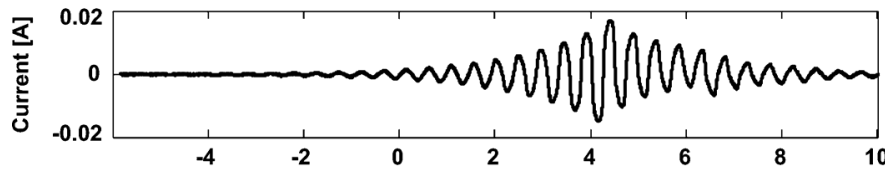

(c)

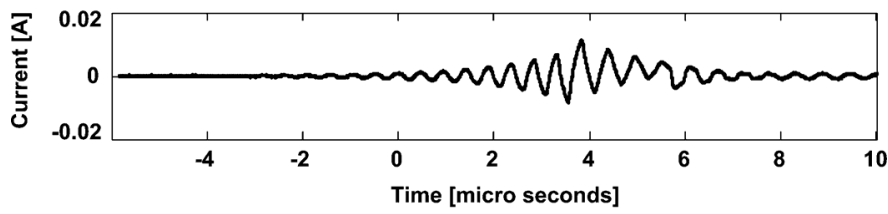

(d)

Fig. 8. Experimentally measured waveforms for a fault location $x_{f}=26 \mathrm{~m}$ for the topology of Fig. 6(a). (a) Direct-time voltage measured at the observation point located at the beginning of the line. Measured fault currents as a result of the injection of time-reversed signal at guessed fault locations $(b) x_{f}^{\prime}=23 \mathrm{~m}$, $(c) x_{f}^{\prime}=26 \mathrm{~m}$ (real fault location) and $(d) x_{f}^{\prime}=28 \mathrm{~m}$.

metal-oxide semiconductor field-effect transistor (MOSFET) (TMS2314) with a turn-on time of 3 ns. The MOSFET was driven by a National Instruments digital input/output (I/O) card C/series 9402 that was able to provide a gate signal to the MOSFET with a subnanosecond rise time and a maximum voltage of $3.4 \mathrm{~V}$. The schematic representation of the circuit of the hardware fault emulator is illustrated in Fig. 7. Note that the experiment reproduces solid faults since no resistors were placed between the MOSFET drain and the transmission-line conductors.

By making reference to the topology of Fig. 6(a), Fig. 8(a) shows the measured direct-time voltage at the considered observation point for a fault location $x_{f}=26 \mathrm{~m}$. The measured voltage was then time-reversed and injected back into the line using the arbitrary waveform generator for each of the 12 different guessed fault locations, that are indicated in Fig. 6(a). For each case, the fault current resulting from the injection of 


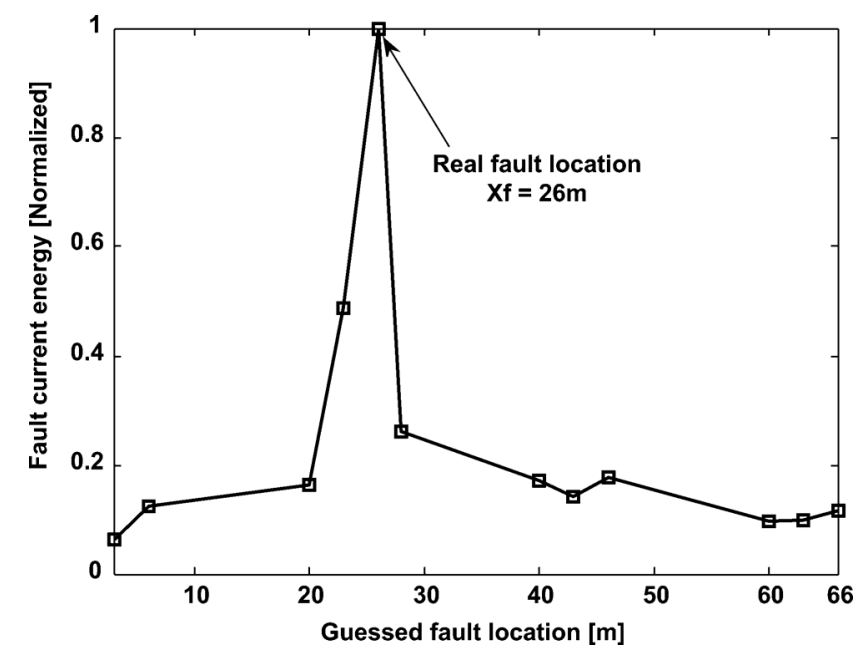

Fig. 9. Normalized energy of the measured fault current as a function of the position of the guessed fault location. The real fault location is $x_{\mathrm{f}}=26 \mathrm{~m}$.

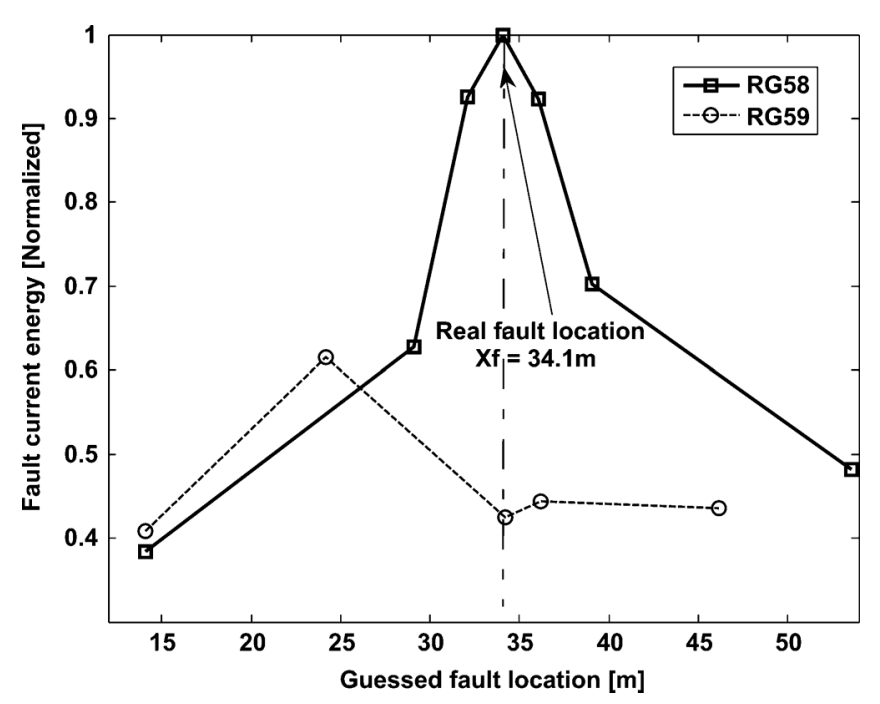

Fig. 10. Normalized energy of the measured fault current as a function of the position of the guessed fault location for the case of the topology presented in Figs. $6(\mathrm{~b})$. The real fault location is $x_{\mathrm{f}}=34.1 \mathrm{~m}$ in RG-58.

the time-reversed signal of Fig. 8(a) was measured using the Pearson current probe. Fig. 8(b)-(d) shows the waveforms of the fault current at the guessed fault locations $x_{f}^{\prime}=23 \mathrm{~m}, x_{f}^{\prime}=$ $26 \mathrm{~m}$, and $x_{f}^{\prime}=28 \mathrm{~m}$, respectively, resulting from the injection of the time-reversed signal.

The normalized energy of the measured fault current signals is shown in Fig. 9 as a function of the guessed fault location (also in this case, the normalization has been implemented with respect to the maximum signal energy of the fault current in the guessed fault location). As can be observed, the correct fault location is uniquely and clearly identified.

Fig. 10 shows the same signal energy profiles for the case of the topology of Fig. 6(b). In this case, the real fault location is at a distance of $34.1 \mathrm{~m}$ from the source and in the RG-58 section of the network. As can be observed, also in the case of a multibranched network with lines characterized by different electrical parameters (i.e., inhomogeneous lines with different

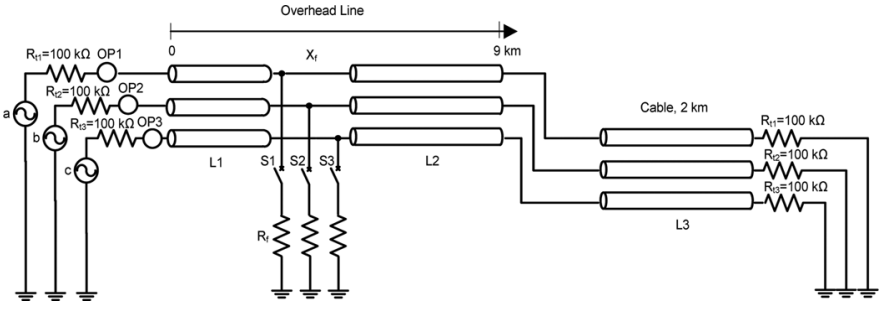

Fig. 11. Schematic representation of the system under study implemented in the EMTP-RV simulation environment.

surge impedances), the proposed methodology correctly identifies the fault location.

\section{APPLICATION EXAMPLES}

\section{A. Inhomogeneous Network Composed of Mixed Overhead-Coaxial Cable Lines}

In this subsection, we present a first numerical validation of the proposed technique. For this purpose, reference is made to the case of a network composed of a three-conductor transmission line and an underground coaxial cable (see Fig. 11).

The overhead line length is $9 \mathrm{~km}$ and the cable length is $2 \mathrm{~km}$. They are modeled by means of a constant-parameter model implemented within the EMTP-RV simulation environment [31]-[33]. Both the overhead line and the cable parameters have been inferred from typical geometries of $230-\mathrm{kV}$ linesand cables. The series impedance and shunt admittance matrices for the line and cable are given by (22)-(25), shown at the bottom of the next page, and have been calculated in correspondence of the line and cable switching frequency. See (22) and (25) at the bottom of the next page.

As can be observed, the simulated lines take into account the losses, even though as discussed in Section III, the EMTR is, in principle, applicable in a lossless propagation medium. However, as already seen in the experimental validation where real cables with losses have been used, the proposed method was still able to effectively locate the fault.

Concerning the line start and cable end, they are assumed to be terminated with power transformers represented, as discussed in the previous sections, by high impedances, assumed, in a first approximation, equal to $100 \mathrm{k} \Omega$. The supply of the line is provided by a three-phase ac voltage source placed at $x=0$. A schematic representation of the system is shown in Fig. 11. All of the fault transients that were observed at the overhead line start in three observation points (OP1, $\mathrm{OP} 2$, OP3) corresponding to the three conductors of the line (left terminal).

Two fault cases are considered to examine the performance of the proposed method for the case of inhomogeneous networks: 1) a three-phase-to-ground fault at $7 \mathrm{~km}$ away from the source with a $0-\Omega$ fault impedance (solid) and 2) a three-phase-toground fault at $5 \mathrm{~km}$ away from the source with a $100-\Omega$ fault impedance (high-impedance fault). In agreement with the proposed procedure, the position of the guessed fault location was moved along the overhead and cable lines assuming, for the fault impedance, apriori fixed values of 1,10 , and $100 \Omega$. It is worth 


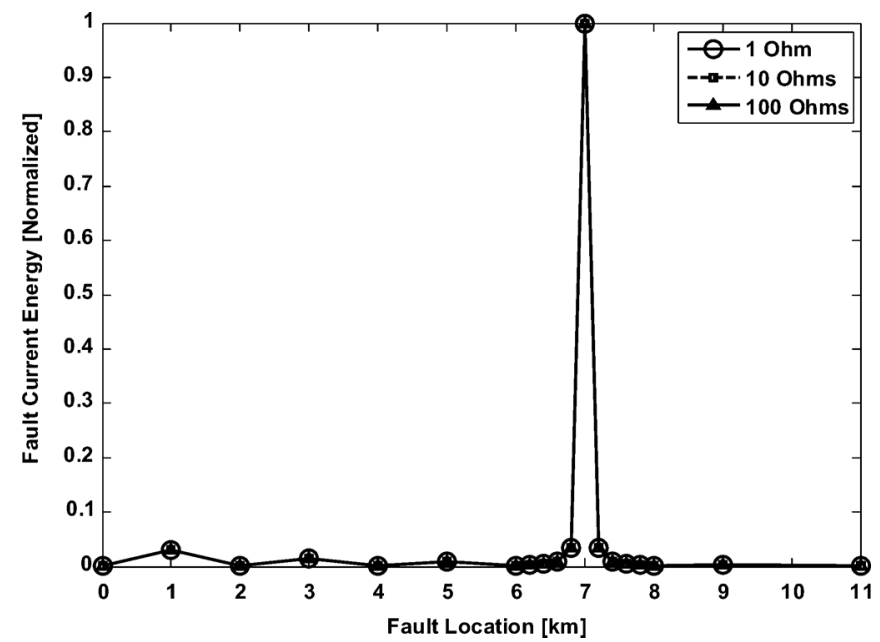

Fig. 12. Normalized energy of the fault current as a function of the guessed fault location and for different guessed fault resistance values. The real fault location is $x_{f}=7 \mathrm{~km}$ and real fault impedance is $0 \Omega$.

noting that in order to find the fault location, it is assumed that the fault type is known form other protective equipment.

Figs. 12 and 13 show the energy of the current flowing through the guessed fault location for solid and high impedance faults, respectively. These figures illustrate the calculated normalized fault current energies for three apriori guessed values of the fault resistance, namely, 1,10 , and $100 \Omega$, as a result of the injection of the time-reversed voltage at the observation points (overhead line left terminal). In order to evaluate the

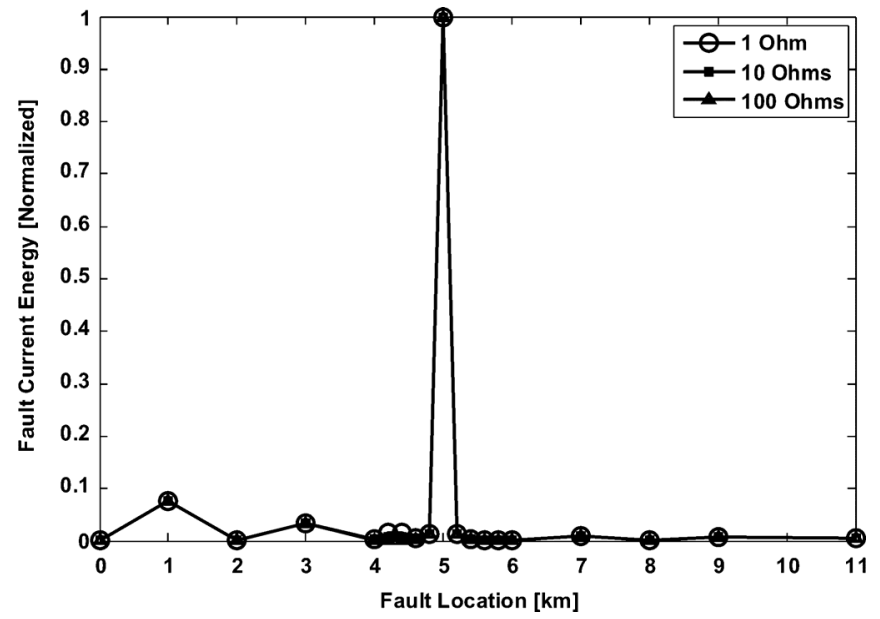

Fig. 13. Normalized energy of the fault current as a function of the guessed fault location and for different guessed fault resistance values. The real fault location is $x_{f}=5 \mathrm{~km}$ and real fault impedance is $100 \Omega$.

accuracy of the proposed method, the position of the guessed fault location was varied with a step of $200 \mathrm{~m}$ close to the real fault location.

As can be seen, the proposed method is effective in identifying the fault location in inhomogeneous networks even when losses are present. The proposed method shows very good performances for high-impedance faults and appears robust against the apriori assumed fault impedance. The accuracy of the method appears to be less than $200 \mathrm{~m}$ (assumed value for the separation between guessed fault locations).

$$
\begin{aligned}
& Z_{\text {Line }}= {\left[\begin{array}{ccc}
1.10+j 15.32 & 1.00+j 5.80 & 1.00+j 4.64 \\
1.00+j 5.80 & 1.09+j 15.33 & 1.00+j 5.80 \\
1.00+j 4.64 & 1.00+j 5.80 & 1.10+j 15.32
\end{array}\right] \frac{\Omega}{\mathrm{km}} } \\
& Y_{\text {Line }}= {\left[\begin{array}{ccccc}
2 \cdot 10^{-4}+j 67.53 & -j 16.04 & -j 7.91 \\
-j 16.04 & 2 \cdot 10^{-4}+j 70.12 & -j 16.04 \\
-j 7.91 & -j 16.04 & 2 \cdot 10^{-4}+j 67.53
\end{array}\right] \times 10^{-6} \frac{\mathrm{S}}{\mathrm{km}} } \\
& Z_{\text {Cable }}= \\
&= \\
&=\left[\begin{array}{ccccccc}
0.07+j 0.70 & 0.05+j 0.45 & 0.05+j 0.41 & 0.05+j 0.62 & 0.05+j 0.45 & 0.05+j 0.41 \\
0.05+j 0.45 & 0.07+j 0.70 & 0.05+j 0.45 & 0.05+j 0.45 & 0.05+j 0.62 & 0.05+j 0.45 \\
0.05+j 0.41 & 0.05+j 0.45 & 0.07+j 0.70 & 0.05+j 0.41 & 0.05+j 0.45 & 0.05+j .62 \\
0.05+j 0.62 & 0.05+j 0.45 & 0.05+j 0.41 & 0.03+j 0.62 & 0.05+j 0.45 & 0.05+j 0.41 \\
0.05+j 0.45 & 0.05+j 0.62 & 0.05+j 0.45 & 0.05+j 0.45 & 0.03+j 0.62 & 0.05+j 0.45 \\
0.05+j 0.41 & 0.05+j 0.45 & 0.05+j 0.62 & 0.05+j 0.41 & 0.05+j 0.45 & 0.03+j 0.62
\end{array}\right] \quad \frac{\Omega}{\mathrm{km}}
\end{aligned}
$$

$Y_{\text {Cable }}=$

$$
\begin{gathered}
=\left[\begin{array}{cccccc}
0.12+j 41.46 & 0 & 0 & -0.12-j 41.46 & 0 & 0 \\
0 & 0.12+j 41.46 & 0 & 0 & -0.12-j 41.46 & 0 \\
0 & 0 & 0.12+j 41.46 & 0 & 0 & -0.12-j 41.46 \\
-0.12-j 41.46 & 0 & 0 & 2.35+j 94.61 & 0 & 0 \\
0 & -0.12-j 41.46 & 0 & 0 & 2.35+j 94.61 & 0 \\
0 & 0 & -0.12-j 41.46 & 0 & 0 & 2.35+j 94.61
\end{array}\right] \\
\times 10^{-6} \frac{\mathrm{S}}{\mathrm{km}} .
\end{gathered}
$$




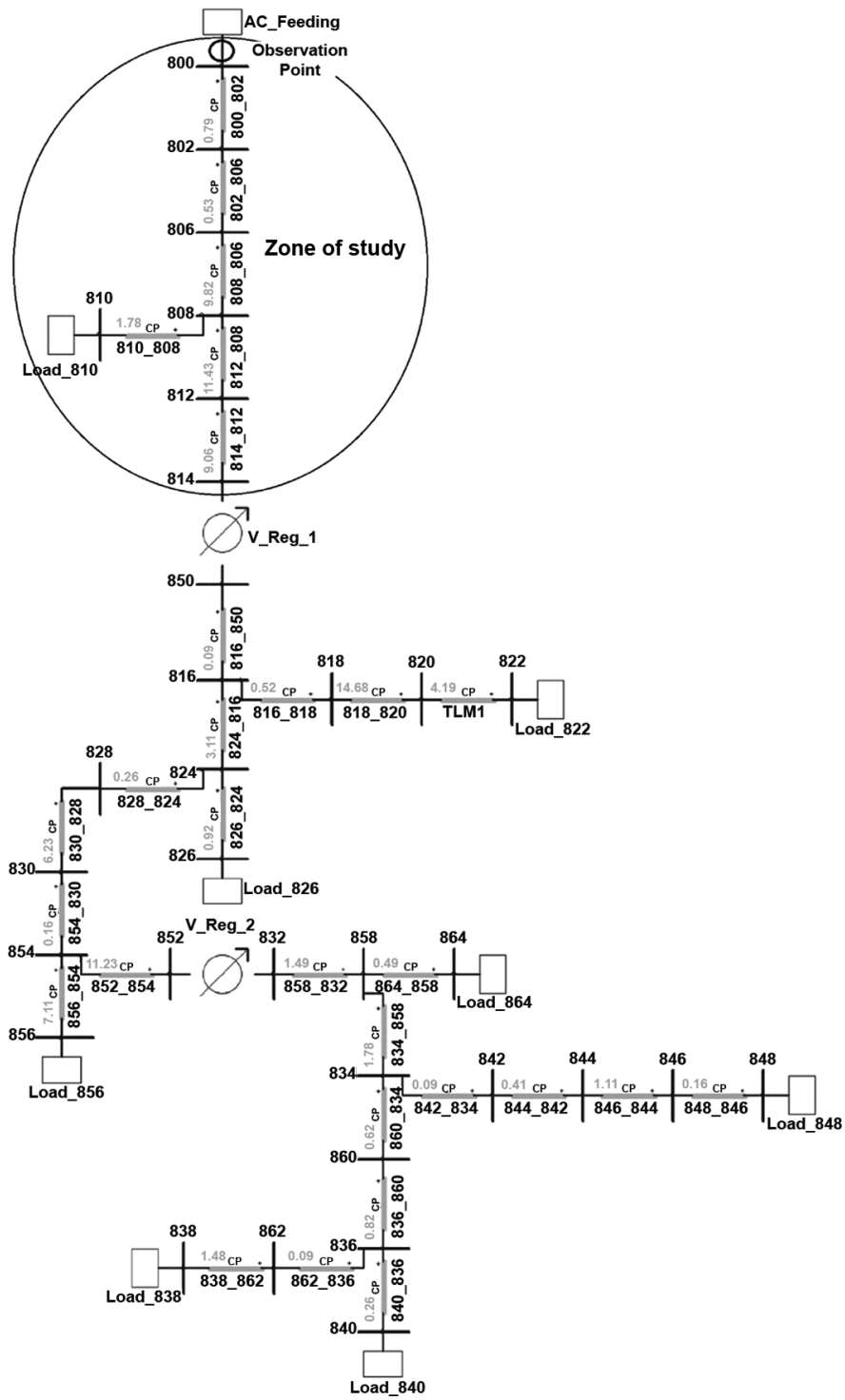

Fig. 14. IEEE 34-bus distribution system implemented in EMTP-RV.

\section{B. Radial Distribution Network: IEEE 34-Bus Test Distribution Feeder}

In order to test the performance of the proposed fault-location method in multibranch and multiterminal networks, the IEEE 34-bus test feeder is considered. The model of this network is the same as that adopted in [13] where, for the sake of simplicity, the following assumptions have been made:

1) all transmission lines are considered to be characterized by configuration "ID \#500" as reported in [34];

2) the loads are considered to be connected via interconnection transformers and are located at lines terminations.

Fig. 14 shows the IEEE 34-bus test distribution network implemented in the EMTP-RV simulation environment. According to the blocking behavior of the transformers for travelling waves, such a configuration could be divided into three zones where these zones are characterized by the buses between two transformers. For this case study, only the first zone is considered as it shown in Fig. 14. The observation point for this network is located at the secondary winding of the transformer and is shown in Fig. 14.

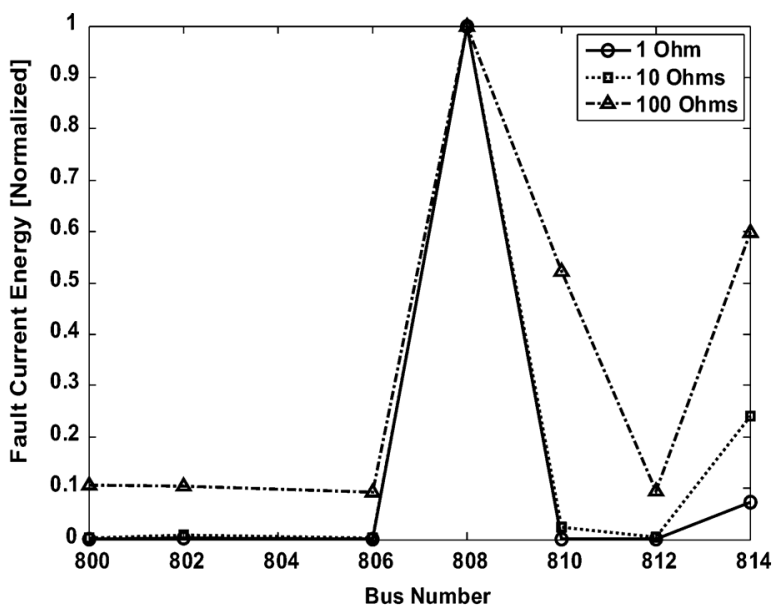

(a)

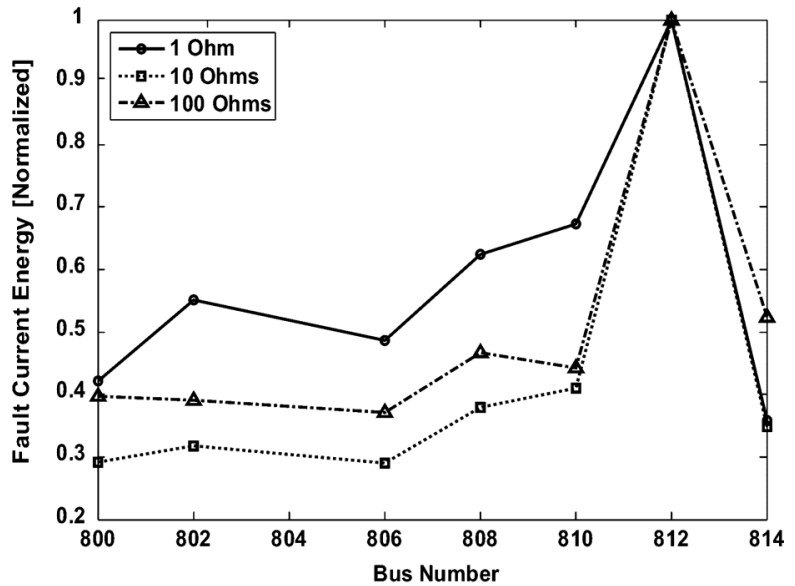

(b)

Fig. 15. Normalized energy of the fault current as a function of the guessed fault location and for different guessed fault resistance values: (a) threephase-to-ground solid fault $(0 \Omega)$ at bus 808 and (b) three-phase-to-ground high-impedance fault $(100 \Omega)$ at bus 812 .

Four different case studies are considered to examine the performance of the proposed method: 1) a three-phase-toground fault at bus 808 with a $0-\Omega$ fault impedance; 2) a three-phase-to-ground fault at bus 812 with a $100-\Omega$ fault impedance; 3 ) a single-phase-to-ground fault at bus 810 with a $0-\Omega$ fault impedance; and 4) a single-phase-to-ground fault at bus 806 with a $100-\Omega$ fault impedance.

The recorded transient signals are time-reversed and, for each guessed fault location, the current flowing through the fault resistance is calculated by simulating the network with back-injected time-reversed signals from the observation points. As for the previous cases, the normalized energy of this current is calculated for all guessed fault locations with different guessed fault impedances (i.e., 1, 10, $100 \Omega$ ). Fig. 15 shows the calculated fault current energy for: 1) a three-phase-to-ground solid $(0 \Omega)$ fault at Bus 808 , and 2) a three-phase-to-ground highimpedance fault $(100 \Omega)$ at bus 812 .

From Figs. 15 and 16, it is possible to infer the remarkable performances of the proposed fault-location method for the case of realistic multibranch multiterminal lines. In addition, the proposed method appears, also in this case, to be robust against solid and high-impedance faults as well as against different fault types (phase-to-ground or three-phase ones). 


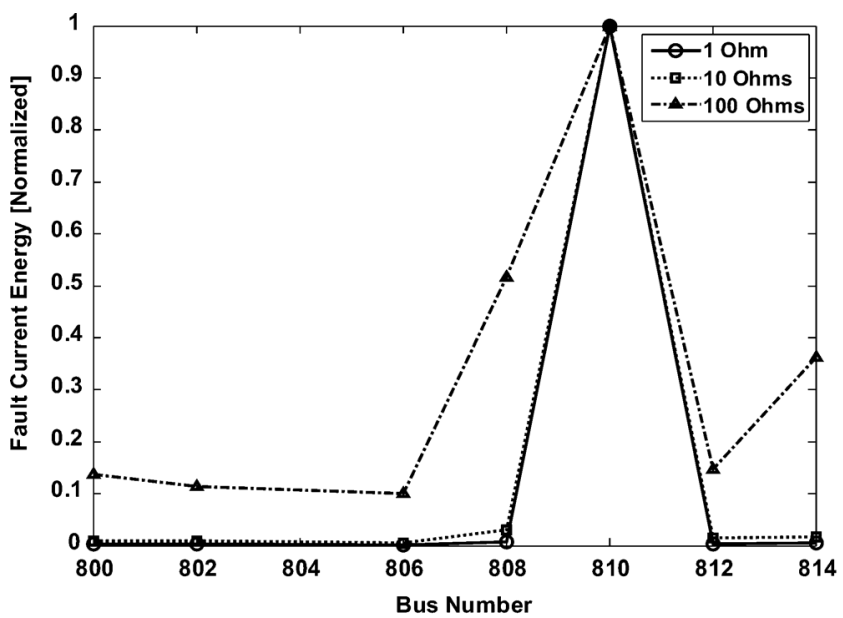

(a)

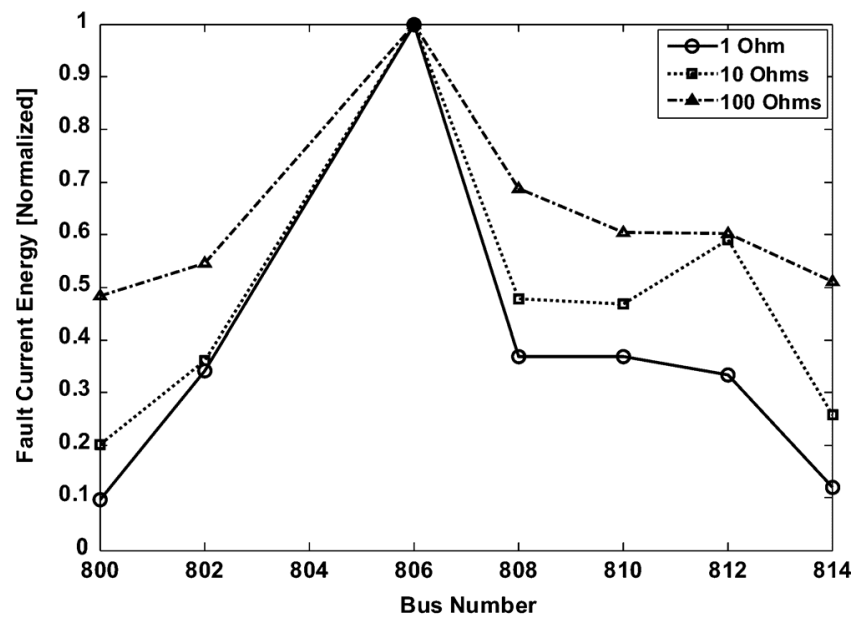

(b)

Fig. 16. Normalized energy of the fault current as a function of the guessed fault location and for different guessed fault resistance values: (a) singlephase-to-ground solid fault $(0 \Omega)$ at bus 810 and (b) single-phase-to-ground high-impedance fault $(0 \Omega)$ at bus 806 .

\section{CONCLUSION}

We presented in this paper a new method to locate faults in power networks based on the use of the EMTR technique. The application of the EMTR to locate faults in a power network is carried out in three steps: 1) measurement of the fault-originated electromagnetic transients in a single observation point; 2) simulation of the back injection of the time-reversed measured fault signal for different guessed fault locations and using the network model; and 3) determination of the fault location by computing, in the network model, the point characterized by the largest energy concentration associated with the back-injected time-reversed fault transients. Compared to other transient-based fault-location techniques, the proposed method is straightforwardly applicable to inhomogeneous media that, in our case, are represented by mixed overhead and coaxial power cable lines. A further advantage of the developed EMTR-based fault-location method is that it minimizes the number of observation points. In particular, the paper has shown that a single observation point located in correspondence of the secondary winding of a substation transformer is enough to correctly identify the fault location.
Another important advantage of the proposed method is that its performances are not influenced by the topology of the system, fault type, and its impedance. The aforementioned peculiar properties have been verified in this paper by applying the proposed method to different networks, namely: 1) a nonhomogeneous network composed of overhead coaxial cable transmission lines and 2) the IEEE distribution test network characterized by multiple terminations.

The proposed method has also been validated by means of reduced-scale experiments considering two topologies, namely, one single transmission line and a T-shape network. In both cases, the proposed EMTR-based approach was able to correctly identify the location of the fault.

The resulting fault-location accuracy and robustness against uncertainties have been tested and, in this respect, the proposed method appears to be very promising for real applications.

\section{ACKNOWLEDGMENT}

The authors would like to thank the anonymous reviewers for their constructive comments that have improved the scientific contents of this paper. The paper takes into account the comments received during the presentation of a preliminary version at the 13th IEEE International Workshop on Signal Processing Advances in Wireless Communications, Cesme, Turkey, June 17-20, 2012.

\section{REFERENCES}

[1] IEEE Guide for Determining Fault Location on AC Transmission and Distribution Lines, IEEE Standard C37.114, 2004.

[2] H. M. Manesh, G. Lugrin, R. Razzaghi, C. Romero, M. Paolone, and F. Rachidi, "A new method to locate faults in power networks based on electromagnetic time reversal," in Proc.13th IEEE Int. Workshop Signal Process. Adv. Wireless Commun., Cesme, Turkey, Jun. 17-20, 2012, pp. 469-474.

[3] M. S. Sachdev and R. Agarwal, "A technique for estimating transmission line fault locations from digital impedance relay measurements," IEEE Trans. Power Del., vol. 3, no. 1, pp. 121-129, Jan. 1988.

[4] K. Srinivasan and A. St.-Jacques, "A new fault location algorithm for radial transmission lines with loads," IEEE Trans. Power Del., vol. 4 no. 3, pp. 1676-1682, Jul. 1989.

[5] A. A. Girgis, D. G. Hart, and W. L. Peterson, "A new fault location technique for two- and three-terminal lines," IEEE Trans. Power Del., vol. 7, no. 1, pp. 98-107, Jan. 1992.

[6] G. B. Ancell and N. C. Pahalawatha, "Maximum likelihood estimation of fault location on transmission lines using travelling waves," IEEE Trans. Power Del., vol. 9, no. 2, pp. 680-689, Apr. 1994.

[7] O. Chaari, M. Meunier, and F. Brouaye, "Wavelets: A new tool for resonant grounded power distribution systems relaying," IEEE Trans. Power Del., vol. 11, no. 3, pp. 1301-1308, Jul. 1996.

[8] F. H. Magnago and A. Abur, "Fault location using wavelets," IEEE Trans. Power Del., vol. 13, no. 4, pp. 1475-1480, Oct. 1998.

[9] F. H. Magnago and A. Abur, "A new fault location technique for radial distribution systems based on high frequency signals," in Proc. IEEE Power Eng. Soc. Summer Meeting, Jul. 18-22, 1999, vol. 1, pp. 426-431.

[10] D. W. P. Thomas, R. E. Batty, C. Christopoulos, and A. Wang, "A novel transmission-line voltage measuring method," IEEE Trans. Instrum. Meas., vol. 47, no. 5, pp. 1265-1270, Oct. 1998.

[11] F. Yan, Zh. Chen, Zh. Liang, Y. Kong, and P. Li, "Fault location using wavelet packets," in Proc. Int. Conf. Power System Technol., Oct. 13-17, 2002, vol. 4, pp. 2575-2579.

[12] A. Borghetti, S. Corsi, C. A. Nucci, M. Paolone, L. Peretto, and R. Tinarelli, "On the use of continuous-wavelet transform for fault location in distribution power networks," Elect. Power Energy Syst., vol. 28, pp. 608-617, 2006.

[13] A. Borghetti, M. Bosetti, M. Di Silvestro, C. A. Nucci, and M. Paolone, "Continuous-wavelet transform for fault location in distribution power networks: definition of mother wavelets inferred from fault originated transients," IEEE Trans. Power Syst., vol. 23, no. 2, pp. 380-388, May 2008. 
[14] A. Borghetti, M. Bosetti, C. A. Nucci, M. Paolone, and A. Abur, "Integrated use of time-frequency wavelet decompositions for fault location in distribution networks: theory and experimental validation," IEEE Trans. Power Del., vol. 25, no. 4, pp. 3139-3146, Oct. 2010.

[15] M. Fink, C. Prada, F. Wu, and D. Cassereau, "Self focusing with time reversal mirror in inhomogeneous media," in Proc. IEEE Ultrasonic Symp., 1989, pp. 681-686.

[16] M. Fink, "Time reversal of ultrasonic fields. I. basic principles," IEEE Trans. Ultrason., Ferroelectr., Freq. Control, vol. 39, no. 5, pp. 555-566, Sep. 1992.

[17] F. Wu, J.-L. Thomas, and M. Fink, "Time reversal of ultrasonic fields II: Experimental results," IEEE Trans. Ultrason., Ferroelect., Freq. Control, vol. 39, no. 5, pp. 567-578, Sep. 1992.

[18] D. Cassereau and M. Fink, "Time-reversal of ultrasonic fields. III. Theory of the closed time-reversal cavity," IEEE Trans. Ultrason., Ferroelect., Freq. Control, vol. 39, no. 5, pp. 579-592, Sep. 1992.

[19] G. Lerosey, J. De Rosny, A. Tourin, A. Derode, G. Montaldo, and M. Fink, "Time reversal of electromagnetic waves," Phys. Rev. Lett., vol. 92, pp. 193901-193904, 2004.

[20] D. Liu, G. Kang, L. Li, Y. Chen, S. Vasudevan, W. Joines, Q. Huo Liu, J. Krolik, and L. Carin, "Electromagnetic time-reversal imaging of a target in a cluttered environment," IEEE Trans. Antennas Propag., vol. 53, no. 9, pp. 3058-3066, Sep. 2005.

[21] H. Zhai, S. Sha, V. K. Shenoy, S. Jung, M. Lu, K. Min, S. Lee, and D. S. $\mathrm{Ha}$, "An electronic circuit system for time-reversal of ultra-wideband short impulses based on frequency-domain approach," IEEE Trans. Microw. Theory Tech., vol. 58, no. 1, pp. 74-86, Jan. 2010.

[22] N. Mora, F. Rachidi, and M. Rubinstein, "Application of the time reversal of electromagnetic fields to locate lightning discharges," $J$. Atmospher. Res., vol. 117, pp. 78-85, 2012.

[23] A. Layane, A. Cozza, and L. Pichon, "A matched-pulse approach for soft-fault detection in complex wire networks," IEEE Trans. Instrum. Meas., vol. 61, no. 6, pp. 1719-1732, Jun. 2012.

[24] J. G. Minonzio, "Décomposition de l'opérateur de retournement temporel appliquée à l'imagerie et à la caractérisation ultrasonore," $\mathrm{Ph} . \mathrm{D}$ dissertation, Univ. Paris 7, Paris, France, 2006.

[25] L. Abboud, A. Cozza, and L. Pichon, "A non-iterative method for locating soft faults in complex wire networks," IEEE Trans. Veh. Technol., vol. 62, no. 3, pp. 1010-1019, Mar. 2013.

[26] G. Lugrin, N. Mora, F. Rachidi, M. Rubinstein, and G. Diendorfer, "On the use of the time reversal of electromagnetic fields to locate lightning discharges," presented at the 31st Int. Conf. Light. Protect., Vienna, Austria, Sep. 3-7, 2012.

[27] A. Greenwood, Electrical Transients in Power Systems AJSA. New York: Wiley, 1991.

[28] A. Borghetti, M. Bosetti, C. A. Nucci, M. Paolone, and A. Abur, "Fault location in active distribution networks by means of the continuous-wavelet analysis of fault-originated high frequency transients," in Proc. CIGRÉ General Session, Paris, France, Aug. 22-27, 2010, paper $\mathrm{C} 4-108$.

[29] F. Rachidi, C. A. Nucci, M. Ianoz, and C. Mazzetti, "Influence of a lossy ground on lightning-induced voltages on overhead lines," IEEE Trans. Electromagn. Compat., vol. 38, no. 3, pp. 250-264, Aug. 1996.

[30] F. Rachidi, "A review of field-to-transmission line coupling models with special emphasis to lightning-induced voltages," IEEE Trans. Electromagn. Compat., vol. 54, no. 4, pp. 898-911, Aug. 2012.

[31] H. W. Dommel, "Digital computer solution of electromagnetic transiens in single and multiphase networks," IEEE Trans. Power App. Syst., vol. PAS-88, no. 4, pp. 388-399, Apr. 1969.

[32] J. Mahseredjian, S. Lefebvre, and X.-D. Do, "A new method for timedomain modelling of nonlinear circuits in large linear networks," in Proc. 11th Power Syst. Comput.Conf., Avignon, France, Aug. 1993, pp. 915-922.

[33] J. Mahseredjian, S. Dennetière, L. Dubé, B. Khodabakhchian, and L. Gérin-Lajoie, "On a new approach for the simulation of transients in power systems," Elect.Power Syst. Res., vol. 77, no. 11, pp. 1514-1520, Sep. 2007.

[34] IEEE Distribution Planning Working Group, "Radial distribution test feeders," IEEE Trans. Power Syst., vol. 6, no. 3, pp. 975-985, Aug. 1991.

Reza Razzaghi (S'10) received the B.Sc. and M.Sc. degrees in electrical engineering from the University of Tehran, Tehran, Iran, in 2009 and 2011, respectively, and is currently pursuing the Ph.D. degree in electrical engineering at the Distributed Electrical System Laboratory (DESL) of the Ecole Polytechnique Fédérale de Lausanne (EPFL), Lausanne, Switzerland.

His research interests include real-time simulation of power systems, electromagnetic transients, as well as power system protection and control.
Gaspard Lugrin received the M.Sc. degree in electrical engineering from the Swiss Federal Institute of Technology of Lausanne (EPFL), Lausanne, Switzerland, in 2011, where he is currently pursuing the Ph.D. degree in electrical engineering at the Electromagnetic Compatibility Group, EPFL.

His research interests include different topics dealing with electromagnetic compatibility, such as electromagnetic coupling to structures and high-power electromagnetics.

Hossein Manesh was born in Iran in 1984. He received the M.Sc. degree in electrical engineering from the University Claude Bernard Lyon 1, Lyon, France, in 2012 and is currently pursuing the Ph.D. degree in electrical engineering at the Paris Electrical Engineering Laboratory (LGEP), University Paris-Sud, Paris, France, as well as at the Electronic, Communication, Systems and Microsystems Laboratory (ESYCOM), University Paris-Est (UPEMLV), France.

During his master's study, he was working with the EMC Group and at the Distributed Electrical System Laboratory (DESL), Swiss Federal Institute of Technology of Lausanne (EPFL), Lausanne, Switzerland, from 2011 to 2012. His current research interests include electromagnetic compatibility and numerical computation of electromagnetic fields.

Carlos Romero received the Ph.D. degree in electrical engineering from the Ecole Polytechnique Fédérale de Lausanne (EPFL), Lausanne, Switzerland, in 2013.

From 2003 to 2008, he was with the National University of Colombia, Bogota, Colombia, and later as Chief of Engineering of the Colombian Satellite Program. He is currently conducting a postdoctoral research project with the EMC Group, EPFL Lausanne, Switzerland, and a private company. His research interests focus on electromagnetics, lightning, and electromagnetic compatibility.

Mario Paolone (M'07-SM'10) was born in Italy in 1973. He received the M.Sc. degree (Hons.) in electrical engineering and the Ph.D. degree in electrical engineering from the University of Bologna, Bologna, Italy, in 1998 and 2002, respectively.

In 2005, he was appointed Researcher in Electric Power Systems, University of Bologna, where he was with the Power Systems Laboratory until 2011. In 2010, he received Associate Professor eligibility from the Politecnico di Milano, Milan, Italy. Currently, he is Associate Professor at the Swiss Federal Institute of Technology of Lausanne (EPFL), Lausanne, Switzerland, where he accepted the EOS Holding Chair of Distributed Electrical Systems Laboratory.

Dr. Paolone is Secretary and Member of several IEEE and CIGRÉ working groups. He was $\mathrm{Co}$-Chairperson of the technical committee of the ninth edition of the International Conference of Power Systems Transients. His research interests are in the areas of smart grids, with particular reference to real-time monitoring and operation, power system protections, power systems dynamics, and power system transients with particular reference to Lightning-Electromagnetic Pulse (LEMP) interaction with electrical networks.

Farhad Rachidi (M'93-SM'02-F'10) received the M.S. degree in electrical engineering and the Ph.D. degree in electrical engineering from the Swiss Federal Institute of Technology of Lausanne (EPFL), Lausanne, Switzerland, in 1986 and 1991, respectively.

He was with the Power Systems Laboratory, EPFL, until 1996. In 1997, he joined the Lightning Research Laboratory, University of Toronto, Toronto, ON, Canada and from 1998 until 1999, he was with Montena EMC, Rossens, Switzerland. Currently, he is the Head of the EMC Laboratory, EPFL, Lausanne, Switzerland. He is the author or coauthor of more than 300 scientific papers published in reviewed journals and presented at international conferences.

Dr. Rachidi was the Vice-Chair of the European COST Action on the Physics of Lightning Flash and its Effects (2005-2009) and the Chairman of the 2008 European Electromagnetics International Symposium (EUROEM). Currently, he is the President of the International Conference on Lightning Protection (ICLP) and Editor-in-Chief of the IEEE TRANSACTIONS ON ELECTROMAGNETIC COMPATIBILITY. In 2005, he was the recipient of the IEEE Technical Achievement Award and the CIGRE Technical Committee Award. He was awarded the 2006 Blondel Medal from the French Association of Electrical Engineering, Electronics, Information Technology, and Communication (SEE). 